\title{
Nodular Lymphocyte Predominant Hodgkin Lymphoma by Ann Arbor Stage
}

National Cancer Institute

\section{Source}

National Cancer Institute. Nodular Lymphocyte Predominant Hodgkin Lymphoma by Ann

Arbor Stage. NCl Thesaurus. Code C141225.

An anatomic stage for nodular lymphocyte predominant Hodg kin lymphoma based on the Ann Arbor classification criteria. 